\title{
A new indoor localization system based on Bayesian graphical model
}

\begin{abstract}
Indoor localization techniques that use wireless local area network beacon signals have recently gained considerable attention among research communities. System accuracy is one of the most important issues in indoor localization technology. We propose a Bayesian graphical model based on fingerprinting location algorithm in this study. The proposed Bayesian model was simulated using OpenBUGS, a graphical user interface. We conducted an experiment to collect a sample of reference points in a testbed with a dimension of $51 \times 22$ $\mathrm{m} 2$. Results show that the proposed model has improved the accuracy by $25.65 \%$ using 15 reference points compared with Madigan model.
\end{abstract}

Keyword: Bayesian graphical model; Fingerprinting technique; Received signal strength 